\title{
Specific Genes Identified in Pathotype 4 of the Clubroot Pathogen Plasmodiophora brassicae
}

Jing Zheng, Xuliang Wang, Yang Xiao, Shiqing Wei, Die Wang, and Yun Huang, College of Agronomy, Sichuan Agricultural University Chengdu Campus, Chengdu 611130, China; Wenming Wang, Rice Research Institute and Research Center for Major Crop Diseases, Sichuan Agricultural University Chengdu Campus, Chengdu 611130, China; and Hui Yang, ${ }^{\dagger}$ College of Agronomy, Sichuan Agricultural University Chengdu Campus, Chengdu 611130, China

\begin{abstract}
Clubroot is an important disease of cruciferous crops caused by Plasmodiophora brassicae, and pathotypes are classified based on the response of differential hosts. This study was conducted to identify genetic markers able to differentiate pathotypes. Differential expression of genes between pathotype 4 (P4) and pathotype 7 (P7) was assessed according to transcriptome data of molecular marker screening. Among the pathotypes (P2, P4, P5, P7, P9, P10, and P11) tested, six genes were exclusive to P4, dividing the isolates into three types: PBRA_003263 and PBRA_

003268 were present in all P4 isolates, PBRA_000003/Novel512 were found in a type of P4 (P4-1), and Novel137/PBRA_005772 were found in another P4 type, P4-2. Amplicons for all six genes were produced for only one isolate, which we named P4-3. This study is the first to establish a molecular identification system for P4 the, predominant pathotype in China. The genes identified might serve as molecular markers for differentiation of $\mathrm{P} 4$ from other pathotypes and may also distinguish different types of $\mathrm{P} 4$.
\end{abstract}

Clubroot is an important disease of cruciferous crops that is caused by Plasmodiophora brassicae Woron, a soilborne, obligate, and biotrophic pathogen that leads to reductions in crop yield (Crisp et al. 1989; Dixon 2009). The disease is spreading rapidly in Asia, becoming a problem not only in China, Japan, and Korea but also in tropical countries such as Indonesia, Thailand, and Vietnam. Clubroot is known to occur in more than 60 countries (Dixon 2009). In China, the disease is present in 28 provinces, causing serious damage to cruciferous crops (Chai et al. 2014).

Clubroot is difficult to control by chemical or cultural means. Many strategies have been proposed to date but the development of resistant cultivars is believed to be the most effective and environmentally friendly solution. Breeding resistant cultivars requires a good understanding of the virulence pattern of pathogen populations, yet the large variation in pathogenicity and the lack of a satisfactory method for detecting specific virulent isolates is a limitation of clubroot control. There are few reports on pathotypes of this pathogen in China. Nonetheless, in efforts to identify pathotypes of $P$. brassicae and accelerate breeding of clubroot-resistant crops, Shen et al. (2009) studied clubroot pathogens from 15 major areas in China (including Liaoning, Jilin, Shandong, and Sichuan Provinces) and discovered that pathotype $4(\mathrm{P} 4)$ was predominant in 11 areas and that $\mathrm{P} 2, \mathrm{P} 7$, $\mathrm{P} 10$, and $\mathrm{P} 11$ consisted of a single isolate.

Because different isolates of $P$. brassicae can only be distinguished by their virulence phenotype, pathotypes have been identified based on the reactions of differential hosts (Buczacki et al. 1975; Kuginuki et al. 1999; Williams 1966). Utilized formally with wide application, the Williams differential system was the first (Williams 1966); in this system, 16 pathotypes can theoretically be identified using four differential hosts. Buczacki et al. (1975) later established the European clubroot differential (ECD) set, which is currently the universal identification system for the clubroot pathogen in Europe. Overall, Williams' differential system and the ECD

${ }^{\dagger}$ Corresponding author: Hui Yang; E-mail: yanghui981@126.com

*The $\boldsymbol{e}$-Xtra logo stands for "electronic extra" and indicates that two supplementary figures and two supplementary tables are published online.

Accepted for publication 5 September 2018.

@ 2018 The American Phytopathological Society set are considered an international identification system for identifying pathotypes of $P$. brassicae.

However, these identification systems have some limitations. For example, the temperature and $\mathrm{pH}$ (Gossen et al. 2013) can influence the activity and pathogenicity of $P$. brassicae and further affect the accuracy of pathotype identification. Pathotype differentiation via virulence tests on differential hosts generally takes as long as 2 months; it is time consuming and labor intensive. Moreover, environmental conditions and various uncertain factors during a long growing period may affect the accuracy of identification (Sharma et al. 2011). Notably, result interpretation is often limited by nonhomogeneous reactions due to the heterogeneity of $P$. brassicae field isolates (Crute and Pink 1989). Therefore, classifying P. brassicae into pathotypes at the molecular level is an important research topic, and the availability of specific molecular markers for particular pathotypes would be valuable for identifying pathotypes and monitoring $P$. brassicae populations. Random amplified polymorphic DNA (RAPD) markers have been developed and applied in pathogen polymorphism analysis (Buhariwalla et al. 1995; Ito et al.1997; Manzanares-Dauleux et al. 2000; Möller and Harling 1996; Yano et al. 1997). For example, Manzanares-Dauleux et al. (2000) obtained a RAPD marker specific to $P$. brassicae P1, as classified based on the differentials of Somé et al. (1996). In addition, Zhang et al. (2015) found that, among 118 nonhousekeeping genes of $P$. brassicae, $\mathrm{Cr} 811$ was exclusively present in $\mathrm{P} 5$ and could distinguish it from P3 and P8 (Zhang et al. 2015). Thus far, molecular identification of $P$. brassicae is challenging due to the limited availability of differentiating polymerase chain reaction (PCR) markers, and molecular markers specific for isolates or groups of pathotypes would accordingly be a valuable tool for $P$. brassicae population identification and monitoring. Establishing a molecular identification system of pathotypes of $P$. brassicae is key to increasing the breeding efficiency of disease-resistant crucifer varieties and achieving better control of clubroot.

Based on current knowledge, the molecular determinants of pathotypes are related to the presence or absence of gene expression. Because differences in pathogenicity might be related to expression of certain genes, it is reasonable to obtain PCR markers according to transcription levels; as such, sequence data are a good resource for marker screening. In this study, transcriptome data of $P$. brassicae on Brassica rapa were analyzed at 35 days postinoculation (dpi). We focused on differences in gene expression between P4 and P7 and further screened molecular markers for specific pathotypes. 
We hypothesized that a portion of the genes identified may be specific to certain pathotypes. The objectives of this study were to identify genes present in certain pathotypes, and the primer pairs specific for these genes were employed to evaluate the presence of the corresponding genes in genomic DNA samples obtained from different pathotypes.

\section{Materials and Methods}

Preparation of resting spore suspension. $P$. brassicae resting spores were prepared as previously described (Ji et al. 2014). Root galls were homogenized in $10 \%$ (wt/vol) sucrose using a blender. The slurry was filtered through eight layers of gauze, and the suspension was clarified by centrifugation at 3,000 rpm for $9 \mathrm{~min}$. The pellet was suspended in $40 \mathrm{ml}$ of sterile water and transferred to a new tube. After centrifugation at $4.000 \mathrm{rpm}$ for $12 \mathrm{~min}$, the resting spore precipitate was resuspended in sterile water, adjusted to a concentration of $1 \times 10^{7}$ spores $/ \mathrm{ml}$, and stored at $4^{\circ} \mathrm{C}$.

$\boldsymbol{P}$. brassicae inoculation. Seed of Chinese cabbage (Zaoshu-5; San Duo Seed) were sterilized with $75 \%$ ethanol for $5 \mathrm{~min}$ and repeatedly washed with sterile water. After the seedlings were 1 week old or the cotyledons were fully expanded, $0.5 \mathrm{ml}$ of the spore suspension prepared as above was inoculated at the base of each seedling. Resting spores were diluted to a density of $10^{7}$ spores $/ \mathrm{ml}$ in sterile distilled water.

Identification of $P$. brassicae isolates by Williams' system. Pathogen populations from different locations in China were classified based on the differentials of Williams (1996), including two cabbage cultivars (Jersey Queen and Badger Shipper) and two rutabaga cultivars (Laurentian and Wilhelmsburger). Seedlings were planted in 4by-4-cm plastic pots, which were filled with a $2: 1$ ratio of soil and peat (pH 6.5) and inoculated with $0.5 \mathrm{ml}$ of spore suspension at a density of $10^{7}$ spores $/ \mathrm{ml}$. The pots were kept in a greenhouse for 45 days; the soil was saturated with water for the first week after inoculation and then fertilized and watered as needed, with the temperature maintained at $24^{\circ} \mathrm{C}$. Ten seedlings of each host line were inoculated with each population of $P$. brassicae; the treatments were replicated three times for a total of 30 plants for each differential per treatment.

Preparation of $B$. rapa with $P$. brassicae inoculation and sequencing. Chinese cabbage plants (Zaoshu-5) were inoculated with a suspension of $P$. brassicae $\mathrm{P} 4$ (isolates DY, SL, and $\mathrm{XC}$ ) or P7 (isolate LX). Resting spores were diluted to a density of $10^{7}$ spores $/ \mathrm{ml}$ in sterile distilled water. The inoculated plants were maintained in a growth chamber under $16 \mathrm{~h}$ of light at $25^{\circ} \mathrm{C}$, and the soil was kept moist during the treatment period. Roots were obtained at 35 dpi and washed thoroughly with distilled water to remove spores adsorbed to the surface, frozen in liquid nitrogen, and stored at $-70^{\circ} \mathrm{C}$. Total RNA was extracted and sent to Novogene Biotechnology Company for sequencing.

RNA-sequencing analysis. RNA was extracted from $P$. brassicae-infected roots samples using TRIzol Reagent (Life Technologies, USA) following the manufacturer's protocol. RNA quantity and quality were determined using a 2100 BioAnalyzer (Agilent Technologies Canada Inc.). RNA libraries were constructed with $2 \mu \mathrm{g}$ of total RNA and subjected to high-throughput sequencing using a HiSeq-4000 sequencer (Illumina). After filtering the raw reads by removing the adapters, reads with 5\% "N", and low-quality reads, clean data were mapped to the reference genome pbe3.h15 by TopHat 2 with default parameters. The reads with an average quality score $>20(\mathrm{Q} 20)$, quality score $>30(\mathrm{Q} 30)$, GC content, and sequence duplication level of the clean data were calculated. The method of fragments per kilobase of transcript per million fragments mapped (FPKM) was used to calculate the normalized expression data for each gene.

We compared expression levels of P4 with P7 as well as expression among different $\mathrm{P} 4$ isolates. To obtain genes that may have associations with P4 identification, we selected those that were highly expressed (FPKM $\geq 5$ ) in P4 but showed low expression (FPKM $<$ 1) in P7. Venn diagrams were used to evaluate the overlap of differentially expressed genes among comparisons using the online tool Venny 2.1 (http://bioinfogp.cnb.csic.es/tools/venny/).

Gene screening and specificity analysis. Chinese cabbage plants (Zaoshu-5) were used as the host to maintain P. brassicae spores. Isolates of LX and KD were classified as P7; DY, SL, LJ, and XC as P4; $\mathrm{LZ}$ as P10; JX as P5; HRB as P2; ES as P11; and NC as P9 according to the identification system of Williams (Supplementary Table S1). Total DNA of root gall samples, including roots and resting spores, were used for the identification of pathotype-specific genes, with screening using select genes based on transcriptome data. Six pathotype-specific genes were obtained and their expression levels for P4-specific identification are listed in Supplementary Table S2. Primer sequences are presented in Table 1. Two pairs of primers for each gene were designed according to the nucleotide sequence. To examine the presence of an intron, transcriptome sequence data were screened using the fragment (Supplementary Fig. S1).

Table 1. Primer sequences used in this research

\begin{tabular}{|c|c|c|c|}
\hline Primer & Sequence & Size (bp) & Annealing temperature $\left({ }^{\circ} \mathrm{C}\right)$ \\
\hline PBRA_003268-F-1 & GAATAGGAAACCCGCCCTGT & 248 & 54 \\
\hline PBRA_003268-R-1 & CGTGATCGTACCAAGACGCA & & \\
\hline PBRA_003268-F-2 & AAGGTCGAACTGCTACACGC & 196 & 54 \\
\hline PBRA_003268-R-2 & CGTGATCGTAAAACCACGCA & & \\
\hline PBRA_003263-F-1 & TTCGCCATATTCCCGGACAA & 214 & 51 \\
\hline PBRA_003263-R-1 & GCATGGAGGACTCGGATTGG & & \\
\hline PBRA_003263-F-2 & ATTCGCCATATTCCCGGACA & 164 & 54 \\
\hline PBRA_003263-R-2 & AGGTCGAACTGCTACACGAA & 366 & 54 \\
\hline PBRA_000003-F-1 & TCGTAGTGCACACTTGCCAT & & \\
\hline PBRA_000003-R-1 & TGACCGGGAAGCTTCGTTAC & & \\
\hline PBRA_000003-F-2 & TAACGAAGCTTCCCGGTCAC & 209 & 54 \\
\hline PBRA_000003-R-2 & AGGGACCATATTCGAACGGC & & \\
\hline Novel512-F-1 & TACTGTACGCGAGCACATGG & 255 & 54 \\
\hline Novel512-R-1 & CAAGCATAAGGGCGTGCAAC & & \\
\hline Novel512-F-2 & GAGTGATGAGCCCGTACTGC & 207 & 54 \\
\hline Novel512-R-2 & AGCTGAGGGGAATTCCTTGG & & \\
\hline Novel137-F-1 & ACGCTGGAACGCATAGAACA & 469 & 57 \\
\hline Novel137-R-1 & CATACGGGTGGATACGGGTG & & \\
\hline Novel137-F-2 & GTCGGAGGCTTACGTAGGTG & 291 & 57 \\
\hline Novel137-R-2 & CACCATCAACAACATCCCGC & & \\
\hline PBRA_005772-F-1 & CGCCTCATGATGTTCGGGAAG & 615 & 57 \\
\hline PBRA_005772-R-1 & GTGCATGCTCCAACAGTACGA & & \\
\hline PBRA_005772-F-2 & TGATCGAGTACCAGTCCGA & 504 & 58 \\
\hline PBRA_005772-R-2 & AGCACTCGTTGACGGCATGC & & \\
\hline
\end{tabular}


In addition, to further analyze the specificity of the above six genes, we employed 36 field isolates, including P4, P7, and P11. Galls caused by natural infection were collected from different fields in China, predominantly in the Sichuan area, and pathogens were identified using Williams' differential hosts. DNA from 21 galls from the same field in Dayi was utilized for PCR to assess homogeneity in a single field. Resting spores of $P$. brassicae were collected from root galls of different crucifer hosts, and resting-spore DNA was subjected to PCR analysis with pathotype-specific genes.

All PCR analyses were performed in a final volume of $10 \mu \mathrm{l}: 5 \mu \mathrm{l}$ of $2 \times$ Taq Master Mix (Takara), $0.5 \mu l$ of each primer, and $0.5 \mu l$ of DNA template. The concentration of DNA was approximately 200 to $300 \mathrm{ng}$. The first amplification cycle consisted of denaturation at $94^{\circ} \mathrm{C}$ for $2 \mathrm{~min} ; 30$ cycles of $94^{\circ} \mathrm{C}$ for $30 \mathrm{~s}$ (denaturation), $55^{\circ} \mathrm{C}$ for $60 \mathrm{~s}$ (annealing), and $72^{\circ} \mathrm{C}$ for $1 \mathrm{~min}$ (extension); and a final extension at $72^{\circ} \mathrm{C}$ for $7 \mathrm{~min}$. In this study, 25, 30, 33, and 40 cycles achieved the best amplification results.

Analysis of primer specificity affected by different hosts. To assess whether the type of host could affect primer specificity, we mixed DNA of $P$. brassicae with the DNA of nine usual hosts: $B$. rapa kinensis, Raphanus sativus L., B. chinensis var. chinensis, B. napus L., B. juncea (L.) Czern. et Coss., B. oleracea L, B. oleracea L. var. botrytis L., Amaranthus tricolor L., and B. campestris L. subsp. chinensis Makino (var. communis Tsen et Lee). This mixture of resting-spore DNA (DY isolate) with DNA from nine different hosts was employed for primer specificity analysis of PBRA_003263, PBRA_003268, PBRA_000003, and Novel512; in contrast, the DNA mixture of the $\mathrm{XC}$ isolate with DNA from nine different hosts was employed for primer specificity analysis of Novel137 and PBRA_005772. A mixture of DNA including P2 (HRB), P5 (JX), P7 (KD and LX), P9 (NC), P10 (LZ), and P11 (ES) was combined with DNA from the nine different hosts for primer specificity analysis of six genes.

DNA extraction of $\boldsymbol{P}$. brassicae resting spores. Resting spores were washed five times in sterile distilled water, dispersed in $100 \mathrm{mM} \mathrm{MgCl}{ }_{2}$ and $200 \mathrm{mM}$ Tris (pH 7.4) buffer, and treated with DNase I $(30 \mu \mathrm{g} / \mathrm{ml})$ for $3 \mathrm{~h}$ at $37^{\circ} \mathrm{C}$ to eliminate host DNA. The solution was centrifuged at $2,500 \times g$ for $5 \mathrm{~min}$, and the pellet was recovered in $5 \mathrm{mM}$ EDTA, $0.5 \%$ sodium dodecyl sulfate, and $10 \mathrm{mM}$ Tris ( $\mathrm{pH} 7.8$ ) buffer containing proteinase $\mathrm{K}$ at $20 \mu \mathrm{g} / \mathrm{ml}$ for $30 \mathrm{~min}$ at $37^{\circ} \mathrm{C}$, and centrifuged at $4,000 \mathrm{rpm}$ for $10 \mathrm{~min}$. The supernatant was discarded, and the resting spores were frozen in liquid nitrogen and stored $-70^{\circ} \mathrm{C}$.

$P$. brassicae DNA was extracted from the above-prepared resting spores using the cetyltrimethylammonium bromide (CTAB) method. DNA of root galls, including plant tissue and resting spores, was also extracted using CTAB. The samples were ground by first freezing in liquid nitrogen; the powder was transferred to 2-ml centrifuge tubes, after which $700 \mu \mathrm{l}$ of $2 \times$ CTAB $(2 \%$ CTAB, $100 \mathrm{mM}$ Tris-HCl [PH 8.0], $20 \mathrm{mM}$ EDTA, and $1.4 \mathrm{M} \mathrm{NaCl}$ ) was added. The samples were mixed by inversion or gentle shaking and stored at $65^{\circ} \mathrm{C}$ for $1 \mathrm{~h}$. Following centrifugation at $12,000 \times g$ for $15 \mathrm{~min}$ to remove insoluble tissue fragments from the lysate, the supernatant was transferred to a fresh tube. An equal volume of phenol-chloroform-isoamyl alcohol (25:24:1) was added, and the sample was centrifuged at $12,000 \times g$ for $15 \mathrm{~min}$. The suspension was transferred to 2-ml centrifuge tubes, and DNA was precipitated by addition of $1 \mathrm{ml}$ of $100 \%$ ethanol per $0.5 \mathrm{ml}$. After incubation at $-70^{\circ} \mathrm{C}$ for at least $1 \mathrm{~h}$, the sample was centrifuged at $12,000 \times g$ for $15 \mathrm{~min}$ at room temperature. The DNA was washed twice with $1 \mathrm{ml}$ of $75 \%$ ethanol, inverting the tubes three to six times, air dried, dissolved in double-distilled $\mathrm{H}_{2} \mathrm{O}$, and stored at $-20^{\circ} \mathrm{C}$.

Semiquantitative reverse-transcription PCR analysis. To investigate gene expression, root samples were collected at 4, 10, 16, and $35 \mathrm{dpi}$, and total RNA was extracted using TRIpure Reagent (Aidlab Biotech), according to the manufacturer's instructions. cDNA synthesis was carried out according to the manufacturer's instructions (Takara) using 3 to $5 \mu \mathrm{g}$ of total RNA. The P. brassicae Actin gene was used as a reference.

Sequence analysis of six genes. The cDNA sequences of Novel512 and Novell37 were obtained from transcriptome data, and opening reading frames (ORFs) were predicted using the online program ORF finder (https://www.ncbi.nlm.nih.gov/orffinder/). The coding sequences of PBRA_003263, PBRA_003268, PBRA_000003, and PBRA_005772 were obtained from the National Center for Biotechnology Information (NCBI) nucleotide website (https://www.ncbi. nlm.nih.gov/nucleotide/). Analysis of protein sequences of Novel512 and PBRA_003268 was performed using the SMART (http://smart. embl-heidelberg.de/) online protein analysis tool.

\section{Results}

RNA-sequencing analysis. We constructed four cDNA libraries, including DY, SL, and XC, which represent $\mathrm{P} 4$, and LX, which represents P7. From 54,109,698 to 73,155,288 clean reads were obtained, ranging from 8.12 to $10.97 \mathrm{G}$. The GC\% content was found to range from 50 to $56 \%$, Q30\% values were all $>92 \%$, and Q20\% values were all $>97 \%$, providing an indication that the accuracy and quality of the sequencing data were sufficient for further analysis. The sequencing data statistics are shown in Table 2.

In summarizing differential results, we realized that some differences between our P4 isolates might exist; we also compared expression in different isolates of $\mathrm{P} 4$ and found $\mathrm{XC}$ to be different from the other two isolates. Approximately 7,000 genes showed an FPKM $\geq$ 5 after infection with each isolate; namely, expression was high. However, the FPKM of 1,000 genes was $<1$ after infection with all isolates. Approximately 100 genes were highly expressed (FPKM $\geq 5$ ) in $\mathrm{P} 4$ but with low expression $(\mathrm{FPKM}<1)$ in P7; these genes were used to further screen specific molecular markers for P4 (Table 3). Genes exhibiting differential expression were also analyzed using a Venn diagram, illustrating the intersections between genes in the different pathotypes or the different isolates of P4. In total, 34 differential genes were shared by all $\mathrm{P} 4$ isolates, and specific genes were also found for XC, DY, and SL isolates (Fig. 1A). More than 100 genes were highly expressed (FPKM $\geq 5$ ) in $\mathrm{XC}$ but lowly expressed $($ FPKM $<1)$ in DY and SL; however, no differential genes between DY and SL met our criteria (Table 3 ). XC versus DY and XC versus SL showed 100 differential genes in common (Fig. 1B). These data indicate different types for P4.

Six genes can distinguish P4 from P2, P5, P7, P9, P10, and P11 isolates. Differential expression of genes between P4 (DY, SL, and $\mathrm{XC}$ ) and P7 (LX) was analyzed according to the transcriptome data. A high expression level (FPKM $\geq 5$ ) in P4 (DY, SL, and XC) but no expression or obvious change $(\mathrm{FPKM}<1)$ in P7 (LX) were selected. Six genes were screened and qualified for ensuing analysis: PBRA_003263, PBRA_003268,PBRA_000003, Novel512, Novel137, and PBRA_005772. The primer pairs for two genes (PBRA_003263 and PBRA_003268) produced amplicons of the expected size using DY, SL, LX, and XC isolate DNA but not that from isolates HRB, JX, KD, LX, NC, LZ, and ES (Fig. 2). Thus, among the tested $P$.

Table 2. Overview of RNA-sequencing results

\begin{tabular}{lcccccc}
\hline Sample $^{\mathbf{a}}$ & Raw reads & Clean reads & Clean bases $(\mathbf{G})$ & Q30 $(\boldsymbol{\%})$ & ${\text { GC content }(\%)^{\mathbf{b}}}^{\text {Mapped ratio }(\boldsymbol{\%})^{\mathbf{c}}}$ \\
\hline DY & $58,900,176$ & $56,279,490$ & $8.44 \mathrm{G}$ & 92.86 & 56.03 & 61.44 \\
XC & $76,511,728$ & $73,155,288$ & $10.97 \mathrm{G}$ & 92.47 & 50.15 & 53.86 \\
LX & $59,500,066$ & $56,944,556$ & $8.54 \mathrm{G}$ & 92.89 & 55.60 & 57.49 \\
SL & $56,949,296$ & $54,109,698$ & $8.12 \mathrm{G}$ & 92.92 & 55.57 & 61.89 \\
\hline
\end{tabular}

a Isolates DY, XC, and SL were identified as pathotype 4 and LX as pathotype 7 according to the Williams system.

$\mathrm{b}$ The percentage of clean reads with a quality score greater than 30 .

${ }^{\mathrm{c}}$ The percentage of reads mapped to transcripts or unigenes in clean reads. 
brassicae, PBRA_003263 and PBRA_003268 were able to distinguish $\mathrm{P} 4$ from $\mathrm{P} 2, \mathrm{P} 5, \mathrm{P} 7, \mathrm{P} 9, \mathrm{P} 10$, and P11. Additionally, two P4 types were detected using the primer pairs for four genes (PBRA_000003, Novel512, Novell37, and PBRA_005772). PBRA_000003 and Novel512 were found in one type of P4 (DY, SL, and LJ) and Novel137 and PBRA_005772 in another type (XC) (Fig. 2). Therefore, we separated these genes into three types: PBRA_003263 and PBRA_003268 for all P4 isolates, PBRA_000003 and Novel512 for one type of P4, and Novel137 and PBRA_005772 for another type of P4. To ensure the accuracy of the PCR results, another primer pair for each gene was designed; amplicons of the expected size were obtained, consistent with the first PCR assay (Fig. 2).

Six genes applied to field samples. The resting spores of 36 field isolates were collected from the mature galls and evaluated by PCR with each primer pair. The primer pairs for PBRA_003263, PBRA_ 003268, PBRA_000003, Novel512, Novel137, and PBRA_005772 resulted in the expected sizes of amplicons when using genomic DNA of P4 but not that of P7 or the other pathotypes (Fig. 3), which indicated that the six genes distinguished P4 from P7 (isolates 5, 19, 27, 28, 29, 30, 33, and 35) and P11 (isolate 23).

When using primer pairs for PBRA_003263 and PBRA_003268, DNA from all P4 isolates produced the expected amplicon sizes. DNA from approximately $85 \%$ of $\mathrm{P} 4$ isolates produced the expected sizes of amplicons when utilizing primer pairs for PBRA_000003 and Novel512, and we named them P4-1, which was the most common type. DNA from only four field isolates resulted in the expected sizes when using primer pairs for Novel137 and PBRA_005772; we termed this P4-2. One isolate's DNA produced the expected size when including all primers, and we called it P4-3, which was the rarest. Therefore, P4 presented three types. Based on comparison of the PCR results, isolate 1 ; isolates $4,17,22$, and 34 ; and isolates 2,3 , $6,7,8,9,10,11,12,13,14,15,16,18,20,21,24,25,26,31$, and 32 differ from each other (Fig. 3).

Among the 34 isolates obtained from Sichuan area, more than $76 \%$ (26 isolates) were found to be $\mathrm{P} 4$; most of the $\mathrm{P} 4$ isolates were $\mathrm{P} 4-1$, three of them were P4-2, and only one was P4-3. According to the result, eight isolates were other pathotypes. These findings indicate that $\mathrm{P} 4$, comprising three types, with $\mathrm{P} 4-1$ being the most abundant, is the predominant pathotype in the Sichuan area. The geographical distribution of the 34 isolates and their pathotypes are illustrated in Figure 4. P4 was mainly distributed around the Chengdu Plain. Other pathotypes were found to be mainly distributed in cold areas such as Xichang, Aba, and Kangding.

In addition, 21 tested field isolates obtained from Dayi were all identified as P4 and further identified as P4-1 (Fig. 5), which was consistent with the identification results for these field isolates.

Table 3. Summary of gene expression comparison for different samples ${ }^{\mathrm{a}}$

\begin{tabular}{|c|c|c|c|c|c|}
\hline Sample & $\begin{array}{l}\text { FPKM } \geq 5 \text { in } \\
\text { two samples }\end{array}$ & $\begin{array}{l}\text { FPKM }<1 \text { in } \\
\text { two samples }\end{array}$ & $\begin{array}{c}1 \leq \mathrm{FPKM}<5 \text { in } \\
\text { two samples }\end{array}$ & $\begin{array}{c}\text { FPKM } \geq 5 \text { in the } \\
\text { former and FPKM }<1 \\
\text { in the latter }\end{array}$ & $\begin{array}{c}\text { FPKM } \geq 5 \text { in the } \\
\text { latter and FPKM }<1 \\
\text { in the latter }\end{array}$ \\
\hline DY versus LX & 7,071 & 1,005 & 1,000 & 76 & 53 \\
\hline SL versus LX & 7,149 & 858 & 851 & 96 & 44 \\
\hline $\mathrm{XC}$ versus LX & 6,981 & 847 & 953 & 128 & 29 \\
\hline $\mathrm{XC}$ versus DY & 6,962 & 812 & 978 & 118 & 61 \\
\hline $\mathrm{XC}$ versus SL & 7,122 & 715 & 950 & 101 & 79 \\
\hline DY versus SL & 7,320 & 999 & 1,078 & 0 & 0 \\
\hline
\end{tabular}

a We compared 10,143 genes for each sample. FPKM = fragments per kilobase of transcript per million fragments mapped. Samples DY, SL, and XC were identified as pathotype 4 and LX as pathotype 7 according to the Williams system of differential hosts.

A $\quad$ XCvsLX

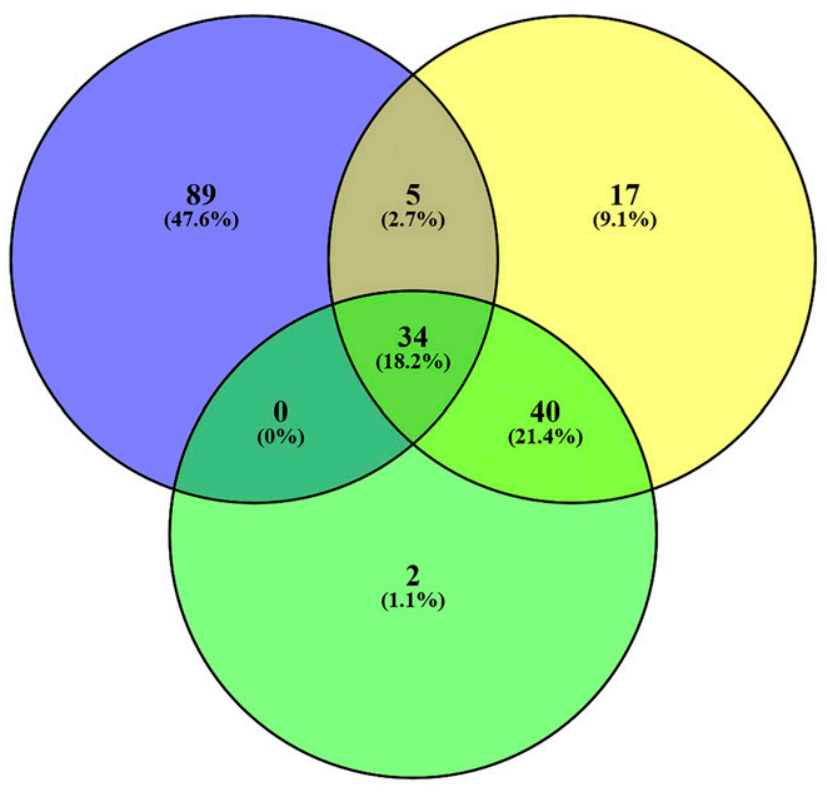

B

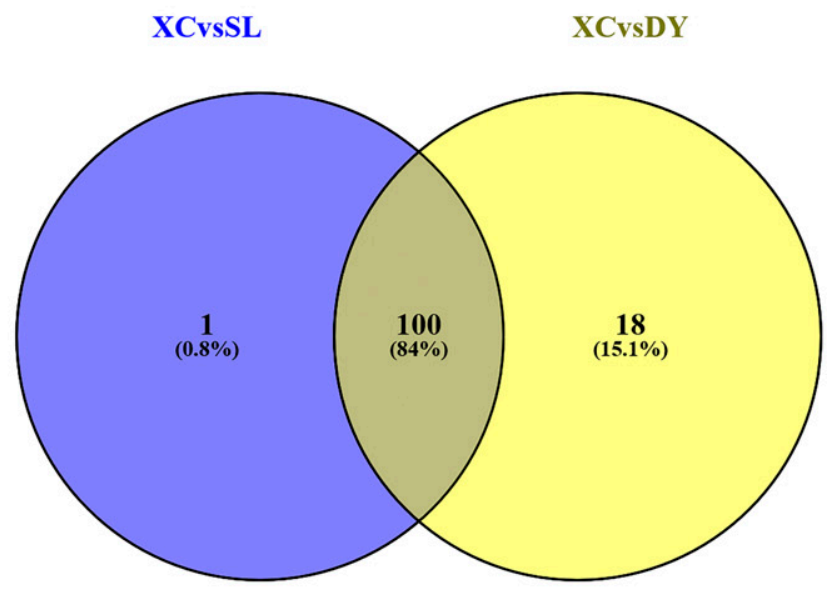

\section{DYvsLX}

Fig. 1. Venn diagram showing the number of differential genes we selected between pathotype 4 (DY, SL, and XC) and pathotype 7 (LX) and different isolates of pathotype 4 . A, Differential genes between pathotype 4 and pathotype 7 and B, differential genes between different isolates of pathotype 4 . Because no differential gene between isolates DY and $\mathrm{SL}$ met our criteria, B only shows differential genes between XC and the other two isolates. 
Specificity analysis of primers for six genes is not affected by the hosts. Because our identification was based on DNA from root galls samples, which included host tissues and resting spores, we sought to determine whether different host tissues might influence PCR results for P4. Primers for PBRA_003263, PBRA_003268, PBRA_000003, and Novel512 specifically amplified target fragments in DNA mixtures of DY isolates and nine different hosts. In contrast, primers for Novel137 and PBRA_005772 produced amplicons when using a DNA mixture of $\mathrm{XC}$ and nine different hosts but did not when using a DNA mixture of P2 (HRB), P5 (JX), P7 (KD and LX), P9 (NC), P10 (LZ), and P11 (ES) and nine different hosts. These results were consistent with the above PCR analysis and indicated that the specificity of these primers for P4 identification is not affected by host DNA (Fig. 6). Thus, a mixture of DNA can be used for identification, without requiring separation of host and pathogen material.

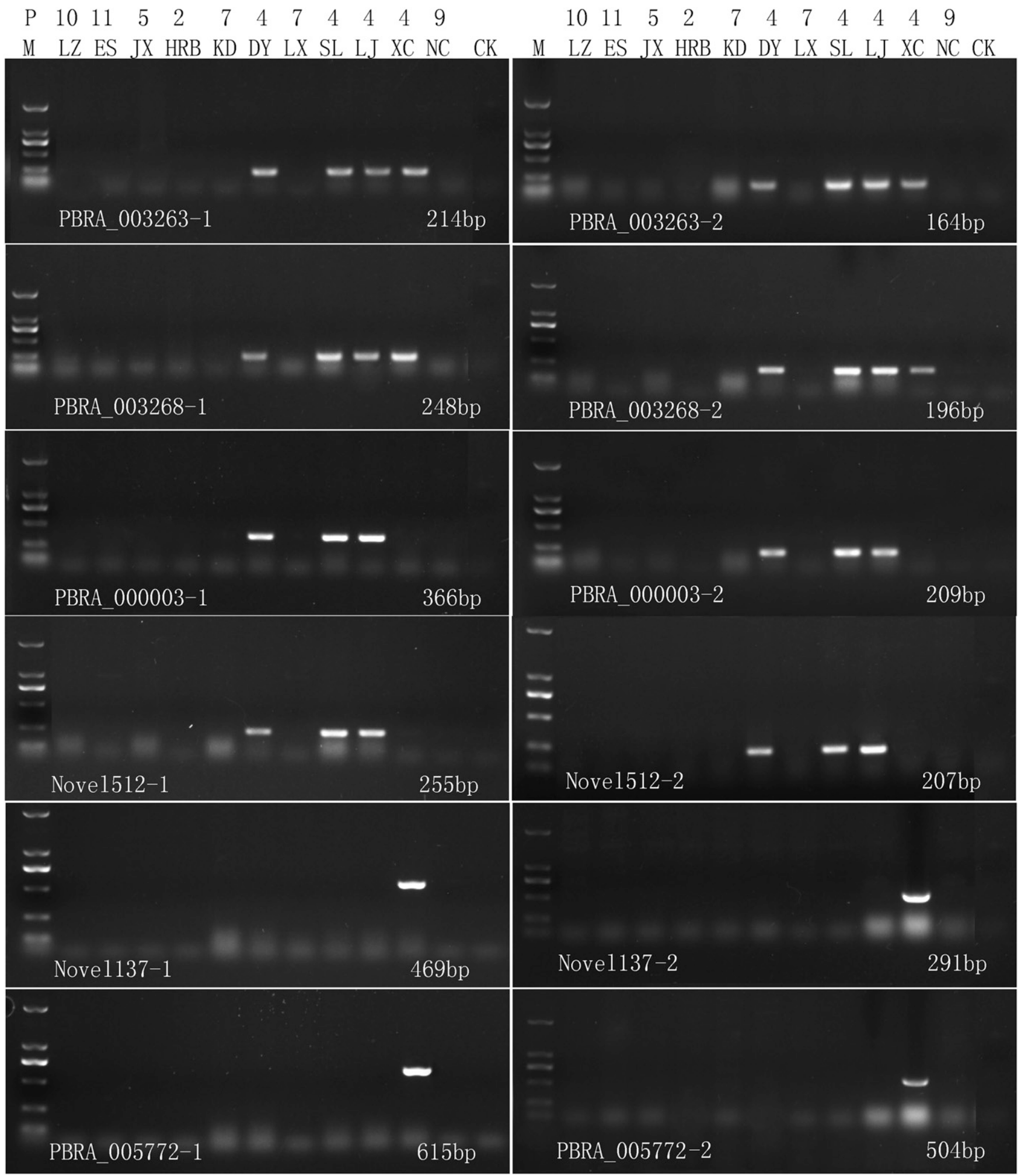

Fig. 2. Identification of the presence of PBRA_003263, PBRA_003268, PBRA_000003, Novel512, Novel137, and PBRA_005772 in different pathotypes of Plasmodiophora brassicae. Isolates DY, SL, LJ, and XC represent pathotype 4 (P4); HRB represents P2; JX represents P5; KD and LX represent P7; NC represents P9; LZ represents P10; and $\mathrm{ES}$ represents $\mathrm{P} 11 . \mathrm{M}=\mathrm{DNA}$ marker $2000, \mathrm{P}=$ pathotype, and $\mathrm{CK}=$ double-distilled $\mathrm{H}_{2} \mathrm{O}$. 
Effect of PCR cycles on P4 identification results. Because different pathotypes, including four dominant races and other rare races, might be simultaneously present in a single gall, complicated results might be obtained when applying molecular identification.
Therefore, four different PCR cycles $(25,30,33$, and 40 cycles) were employed to assess whether rare races present in root gall could affect identification results. We tested six genes based on resting spore DNA extraction of 15 field isolates. Target amplicons were only

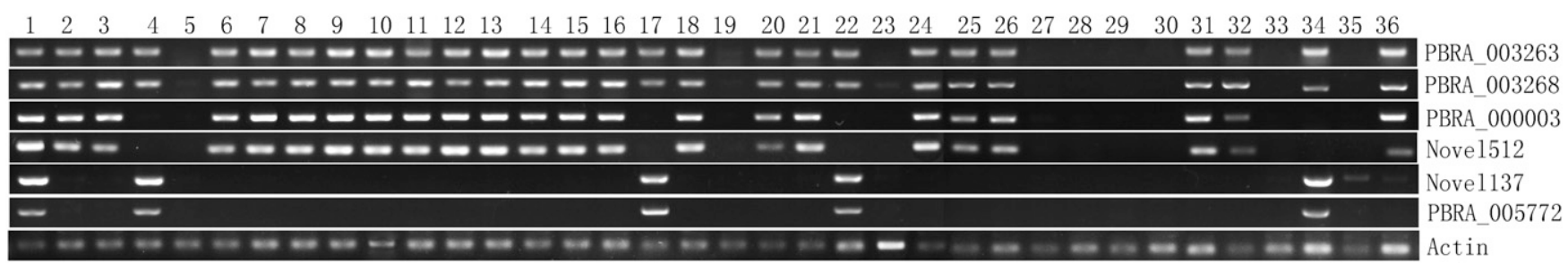

Fig. 3. Pathotype identification of Plasmodiophora brassicae in 36 field isolates. The $P$. brassicae Actin gene was used as a reference gene.

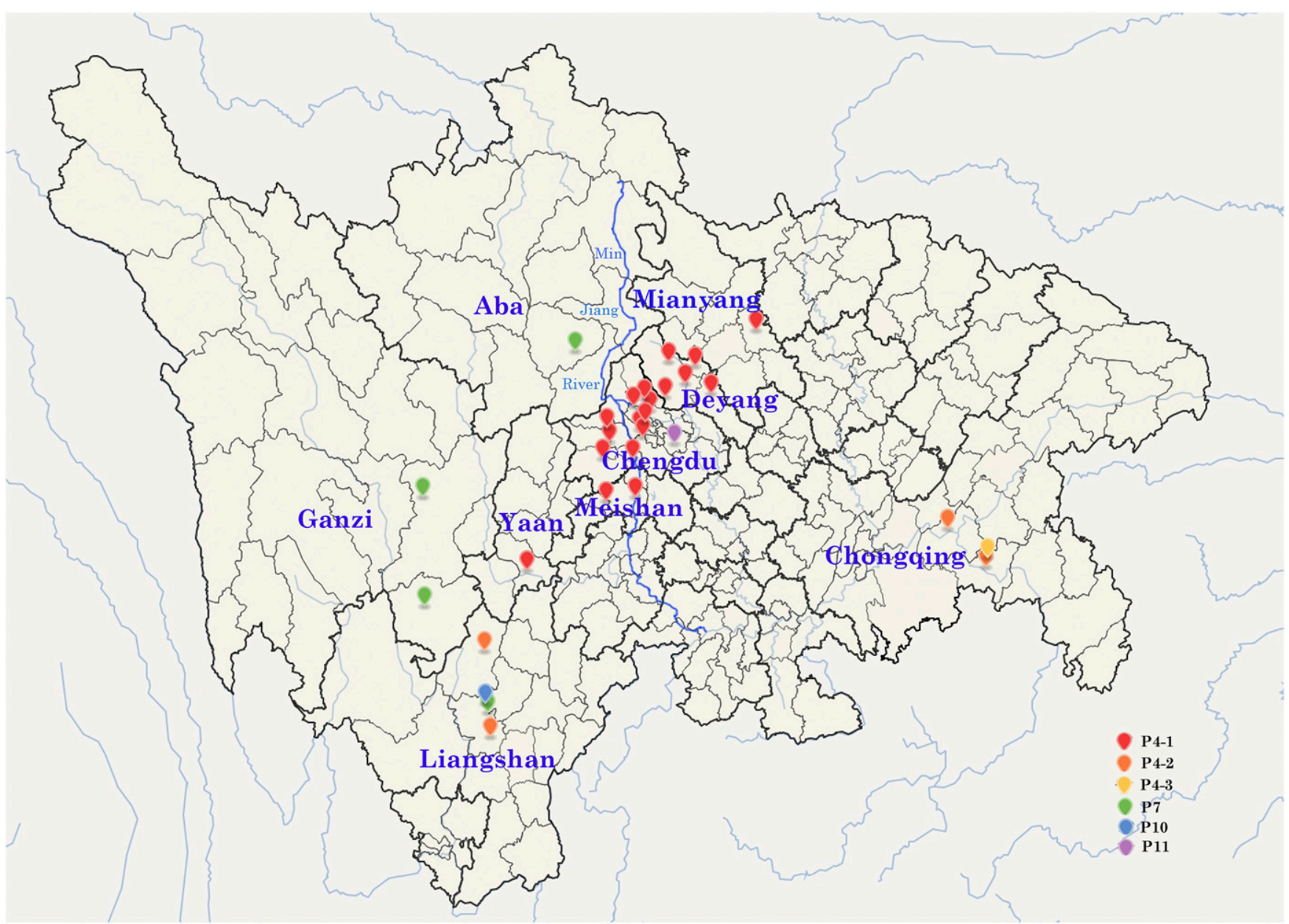

Fig. 4. Identification and distribution of Plasmodiophora brassicae pathotypes in Sichuan Province and Chongqing City.

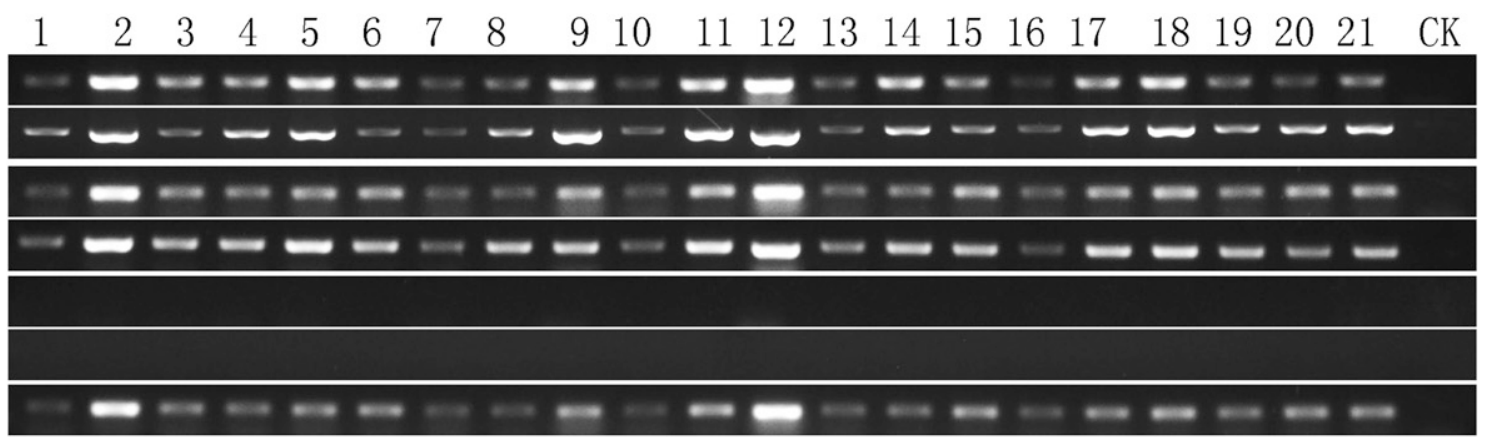

PBRA_000003

PBRA_003263

PBRA_003268

Nove100512

Nove 100137

PBRA_005772

Fig. 5. Pathotype identification of 21 field isolates from Dayi. $\mathrm{CK}=$ double-distilled $\mathrm{H}_{2} \mathrm{O}$. The Plasmodiophora brassicae Actin gene was used as the reference. 
obtained from the corresponding pathotypes with 25 and 30 cycles (Fig. 7). However, target amplicons from noncorresponding pathotypes could also be obtained between 33 and 40 cycles but the amplification level was lower. For example, the expected PCR fragments were more obvious for P4 (isolates $2,4,13,22,25,31,32,34$, and 36) than $\mathrm{P} 7$ (isolates 5, 23, 27, and 35) when using the primers for PBRA_008439 (Fig. 7). At 40 cycles, the amplification level was almost the same among all tested isolates. This result indicated that a mixture of different pathotypes was likely present in a clubroot and that this could be easily confirmed by PCR detection. Therefore, to overcome the disadvantage effect of rare races, the optimal number of PCR cycles should be between 25 and 30 cycles for the identification of P4.

Expression of the six genes during different infection periods. The expression levels of Novel512 were visible at $4 \mathrm{dpi}$, an early stage of infection, and were maintained at the same level at 10 and $16 \mathrm{dpi}$; after which, a peak at $45 \mathrm{dpi}$, during the symptom formation period, was reached. PBRA_003268 was highly expressed at 4 dpi, decreasing slightly at $10 \mathrm{dpi}$, becoming upregulated at later time points, and reaching a peak at $45 \mathrm{dpi}$. For other genes, including PBRA_003263, PBRA_000003, Novel137, and PBRA_005772, the expression levels were hardly observed until they were obvious at 35 dpi (Fig. 8). The six genes were all strongly expressed at 35 dpi, and the expression level of Novel512 and PBRA_003268 was higher than that of the other genes at early time points.

Functional prediction of the six genes. The protein sequences of PBRA_000003, PBRA_003263, PBRA_003268, and PBRA_005772 were obtained from NCBI; all are hypothetical proteins, with protein IDs of CEO94218.1, CEP03656.1, CEP03637.1, and CEO94218.1, respectively. The cDNA sequences of Novel512 and Novel137 were obtained from the transcriptome sequence data. Using ORF finder software, a 174-bp coding sequence was predicted for Novell37 and a 213-bp coding sequence for Novel512. A Blastx search was conducted. The results for Novel137 showed $42 \%$ identity and an $\mathrm{E}-\mathrm{value}=8 \mathrm{e}-5$ for a hypothetical protein (AIF16490.1) from uncultured marine group II/III euryarchaeote KM3_74_D01; for

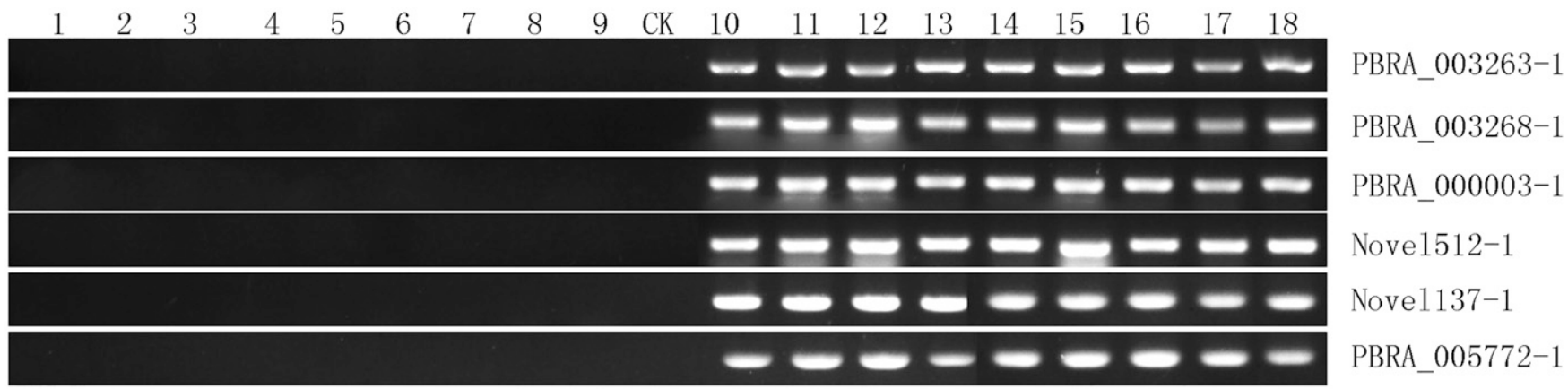

Fig. 6. Analysis of primers specificity affected by different hosts. Lanes A10 to $18, B 10$ to $18, C 10$ to 18 , and D10 to 18 represent DNA mixtures of the DY isolate and nine hosts, respectively. Lanes E10 to 18 and F10 to 18 represent DNA mixtures of the XC isolate and nine hosts, respectively. Lanes 1 to 9 represent a mixture of DNA including pathotype 2 (P2) (HRB), P5 (JX), P7 (KD and LX), P9 (NC), P10 (LZ), and P11 (ES) mixed with DNA from nine different hosts. Hosts (lanes 1 to 9 lanes 10 to 18) were Brassica rapa kinensis, Raphanus sativus L., B. chinensis var chinensis, B. napus L., B. juncea (L.) Czern.et Coss., B. oleracea L, B. oleracea L. var. botrytis L., Amaranthus tricolor L., and B. campestris L. subsp. chinensis Makino (var. communis Tsen et Lee), respectively. $\mathrm{CK}=$ double-distilled $\mathrm{H}_{2} \mathrm{O}$.

PBRA_003268-1

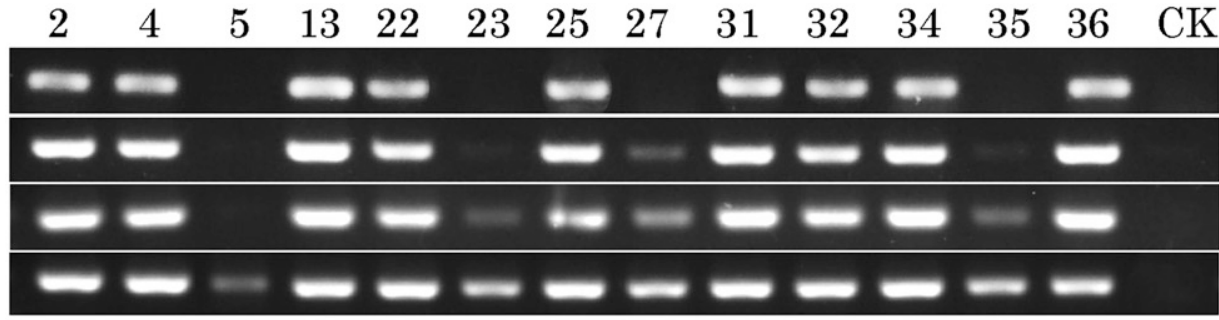

25 cycles 30 cycles 35 cycles 40cycles

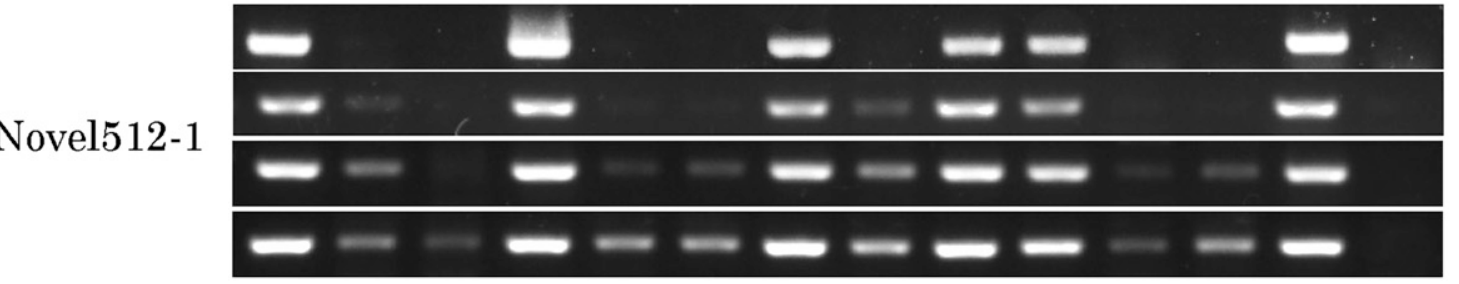

25 cycles 30cycles 35cycles 40cycles

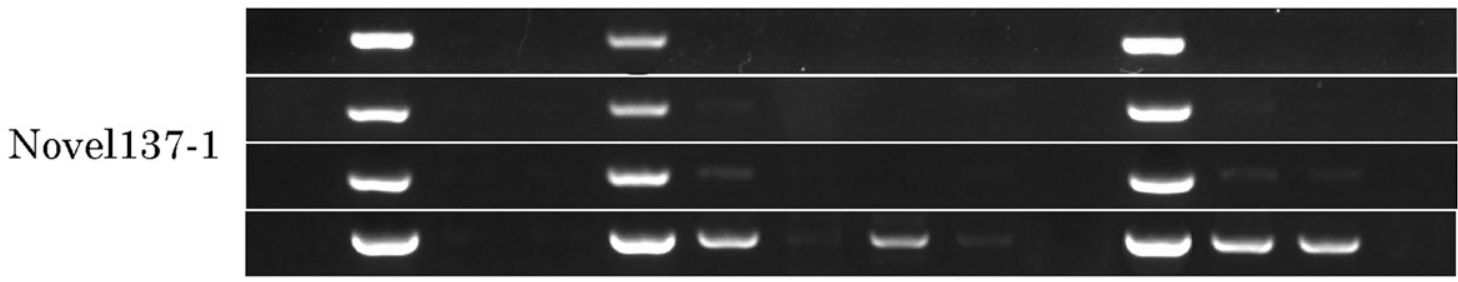

25 cycles 30cycles 35 cycles 40cycles

Fig. 7. Results of different polymerase chain reaction cycles for identification of pathotypes using primers for Novel512, PBRA_003268, and Novel137. Lanes 5 , 27, and 35 represent pathotype 7 (P7); 23 represents P11; and 2, 4, 13, 22, 25, 31, 32, 34, and 36 represent P4. 
Novel512, $42 \%$ identity and E-value $=0.36$ was found for polyketide synthase (NP216043.1) from Mycobacterium tuberculosis H37Rv. A YqgFc domain was identified in PBRA_003268; this domain is present in toxin expression proteins, which are highly conserved bacterial proteins involved in expression of critical toxin genes, though the functions of eukaryotic proteins possessing this domain are not well described.

\section{Discussion}

We conducted RNA sequencing of $\mathrm{P} 4$ and $\mathrm{P} 7$ of $P$. brassicae and selected six genes suitable for P4 identification. No single-spore isolate was used. This approach was rapid and convenient, allowing screening of genes for pathotype identification to select genes present in certain pathotypes but not in others. Indeed, a single gall may harbor different pathotypes but still have a predominant one. Transcriptome analysis has previously been employed to study differential expression of genes during infection stages and gall formation (Chen et al. 2016; Siemens et al. 2006). In the present study, we aimed to identify genes able to discriminate P4, and genes highly expressed $(\mathrm{FPKM} \geq 5)$ in P4 but with low expression $(\mathrm{FPKM}<1)$ in P7 were selected. Ultimately, six genes (PBRA_003263, PBRA_003268, PBRA_000003, Novel512, Novel137, and PBRA_005772) were used to identify different $\mathrm{P} 4$ types. However, whole-genome sequencing of P. brassicae did not identify Novel137 and Novel512, suggesting their absence from in the sequenced strain (Schwelm et al. 2015). Interestedly, PBRA_003263 and PBRA_003268 were found to be present in all P4 isolates examined, PBRA_000003 and Novel512 were present in one P4 type (P4-1), and PBRA_000003 and Novel512 were found in another (P4-2), as confirmed by transcriptome data.

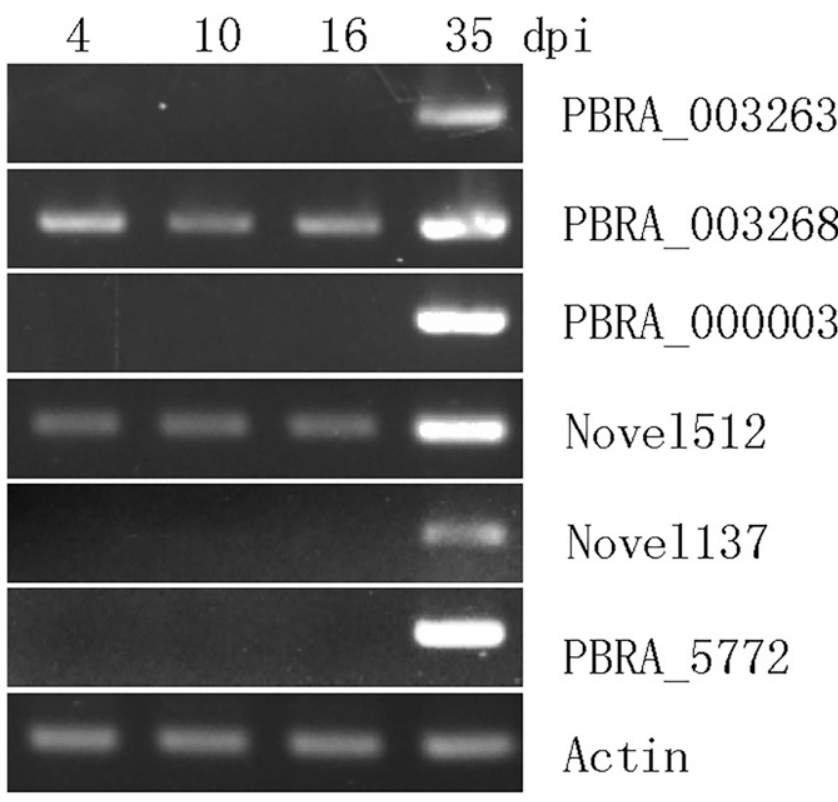

Fig. 8. Expression of PBRA_003263, PBRA_003268, PBRA_000003, Novel512, Novel137, and PBRA_005772 during different infection periods; dpi $=$ days postinoculation. Actin was used as a reference gene.

Table 4. Identification of pathotype 4 (P4) and different types of P4 using different molecular markers ${ }^{\mathrm{a}}$

\begin{tabular}{lcccc}
\hline Gene & P4-1 & P4-2 & P4-3 & Other tested pathotypes \\
\hline PBRA_003263 & + & + & + & - \\
PBRA_003268 & + & + & + & - \\
PBRA_000003 & + & - & + & - \\
Novel512 & + & - & + & - \\
Novel137 & - & + & + & - \\
PBRA_005772 & - & + & + & - \\
\hline
\end{tabular}

a Symbols: + indicates that the target gene could be amplified and - indicates that the target gene could not be amplified.
Based on differences in virulence, various sets of differential hosts have been used to classify $P$. brassicae populations, though this approach has limitations. For example, only 13 pathotypes have been identified to date, indicating that this system is not appropriate for identifying some pathotypes. Various molecular approaches have been developed to improve the practicality of $P$. brassicae pathotype identification. For instance, restriction fragment length polymorphism fingerprinting with highly repetitive elements and RAPD have primarily been applied to characterize isolates of $P$. brassicae (Buhariwalla et al. 1995; Klewer et al. 2001; Möller and Harling 1996; Yano et al. 1997). The RAPD marker OPL14 1200 was found in the molecular pattern of all isolates belonging to one particular pathotype (P1) (Manzanares-Dauleux et al. 2000). In addition, Zhang et al. (2015) used 118 nonhousekeeping genes of $P$. brassicae to evaluate the presence of the corresponding genes in isolates, finally designating $\mathrm{Cr} 811$ presented in P5 and also in P5-like and P3-like. These reports show that PCR markers are suitable for distinguishing among isolates (Faggian et al. 1999; Klewer et al. 2001). Regardless, due to the limitations of differentiating PCR markers, differential classification of pathotypes on a molecular basis is largely unfeasible.

One notion accepted by most researchers is that differences between pathotypes are associated with their genetic makeup, either the absence or presence of certain pathogenicity-related genes or differential expression of those genes (Zhang et al. 2015). Among P2, P4, P5, P7, P9, P10, and P11, we found PBRA_003263, PBRA 003268, PBRA_000003, Novel512, Novel137, and PBRA_005772 to be present in P4 but not in the other pathotypes. PBRA_000003/ Novel512 and Novel137/PBRA_005772 was used to detect two different types identified as $\mathrm{P} 4$ based on Williams' system, indicating a potential method for identifying new strains of $P$. brassicae. Overall, the PCR assay designed in this study will become a valuable tool for differentiating pathotypes. These results indicate that these six genes have the potential to serve as markers to distinguish pathotypes of P. brassicae. PBRA_000003/Novel512 and Novel137/PBRA_ 005772 may be specific molecular markers for different types of P4; the amplification results are summarized in Table 4. Therefore, identification of $\mathrm{P} 4$ and types of $\mathrm{P} 4$ can be achieved using one gene or several genes.

The molecular identification results and geographical distribution of the pathotypes in Sichuan Province and Chongqing City (Fig. 4) were consistent with the previous identification by Peng and Huang et al. (2016) in our lab, as based on Williams' system (unpublished data). Therefore, our PCR assay should also be useful for identifying and monitoring the distribution of $P$. brassicae pathotypes in Sichuan Province. Nonetheless, the results should be confirmed by testing a larger collection of isolates with a broader geographical region and more pathotypes. We also analyzed pathotypes of 21 isolates from the one Dayi field (Fig. 6), all of which were identified as P4-1. These findings indicate that this pathotype is highly conserved in that area and that the molecular markers we obtained are stable and suitable for pathotype identification of $P$. brassicae field isolates.

The occurrence period of $P$. brassicae in these cities and in Sichuan Province differ. According to previous survey sand records, clubroot occurred earlier in Yaan (in 1988) and Chengdu (in 1989) than in other cities. In our study, P4-1 was mainly detected in those areas and around these cities, which might be due to the extensive river system of the Sichuan area. The Jialing River, Minjiang River, and Tuojiang River flow through the Sichuan Basin, which might facilitate the long-distance spread of P. brassicae. P4-2 and P4-3 were only detected in a cold mountain area, which might indicate evolutionary pressure on $\mathrm{P} 4$, whereby an unsuitable environment drove nucleotide sequence changes, producing a different type. P7 was also mainly detected in a cold mountain area, which might have a connection with the local cold climate and cultivated species. Because the water system of the mountain area is not as extensive as that of the plain, we suggest that the spread of the pathogen in those areas mainly depends on human factors such as spread mediated by vehicles.

Overall, the greatest advantage of this PCR method is that it can be performed rapidly and directly from clubroots without purification of 
resting spores. Because host DNA does not affect the results, the technique may provide molecular markers for identifying pathotypes using total DNA from a tiny gall. However, because a mixture of different pathotypes might exist in a root gall, as reported by Somé et al. (1996) and Kuginuki et al. (1999), the number of PCR cycles should be the minimum required. By adding cycles to amplify other pathotypes, our study also confirmed that a field sample is likely a mixture. Therefore, the use of molecular markers to ascertain the presence of pathotypes is fast, with clear results, and the predominant pathotype can also be identified. The use of such markers is underscored by the virulence of populations on all the available commercial canola cultivars, rendering the approach also very useful for breeding and selecting the best resistant cultivar.

Selection of certain pathotypes by host plants has implications for agricultural practice because the infection potential in a certain field depends on the varieties and wild crucifers cultivated in the field. Pathogens and their hosts can respond to changes that occur in each other, and new pathotypes will be generated by mutations in one or some key pathogenic genes, a process that is accelerated in cultivars (as opposed to wild accessions). Thus, molecular markers may be used to gain a better understanding of the genetic variability and structuring factors within populations of $P$. brassicae. Because more molecular markers of this biotrophic protist are expected to be found, future studies should include a larger number of isolates derived from different hosts and geographic origins to find specific markers for different pathotypes.

\section{Literature Cited}

Buczacki, S. T., Toxopeus, H., Mattusch, P., Johnston, T. D., Dixon, G. R., and Hobolth, L. A. 1975. Study of physiological specialization in Plasmodiophora brassicae: Proposals for attempted rationalization through an international approach. Trans. Br. Mycol. Soc. 65:295-303.

Buhariwalla, H., Greaves, S., Magrath, R., and Mithen, R. 1995. Development of specific PCR primers for amplification of polymorphic DNA from the obligate root pathogen Plasmodiophora brassicae. Physiol. Mol. Plant Pathol. 47:83-94.

Chai, A. L., Xie, X. W., Shi, Y. X., and Li, B. J. 2014. Research status of clubroot (Plasmodiophora brassicae) on cruciferous crops in China. Can. J. Plant Pathol. 36:142-153.

Chen, J. J., Pang, W. X., Chen, B., Zhang, C. Y., and Piao, Z. Y. 2016. Transcriptome analysis of Brassica rapa near-isogenic lines carrying clubrootresistant and -susceptible alleles in response to Plasmodiophora brassicae during early infection. Front. Plant Sci. 6:1183.

Crisp, P., Crute, I. R., Sutherland, R. A., Angell, S. M., Bloor, K., Burgess, H., and Gordon, P. L. 1989. The exploitation of genetic resources of Brassica oleracea in breeding for resistance to clubroot Plasmodiophora brassicae. Euphytica 42:215-226.

Crute, I. R., and Pink, D. A. C. 1989. The characteristics and inheritance of resistance to clubroot in Brassicae oleracea. Asp. Appl. Biol. 23:57-60.

Dixon, G. R. 2009. The occurrence and economic impact of Plasmodiophora brassicae and clubroot disease. J. Plant Growth Regul. 28:194-202.
Faggian, R., Bulman, S. R., Lawrie, A. C., and Porter, I. J. 1999. Specific polymerase chain reaction primers for the detection of Plasmodiophora brassicae in soil and water. Phytopathology 89:392-397.

Gossen, B. D., Kasinathan, H., Cao, T., Manolii, V. P., Strelkov, S. E., Hwang, S. F., and McDonald, M. R. 2013. Interaction of $\mathrm{pH}$ and temperature affect infection and symptom development of Plasmodiophora brassicae in canola. Can. J. Plant Pathol. 35:294-303.

Ito, S., Maehara, T., and Tanaka, S. 1997. Cloning of a single-copy DNA sequence unique to Plasmodiophora brassicae. Physiol. Mol. Plant Pathol. 50:289-300.

Ji, R., Zhao, L., Xing, M., Shen, X., Bi, Q., Peng, S., and Feng, H. 2014. Infection of Plasmodiophora brassicae in Chinese cabbage. Genet. Mol. Res. 13: 10976-10982.

Klewer, A., Luerûen, H., Graf, H., and Siemens, J. 2001. Restriction fragment length polymorphism markers to characterize Plasmodiophora brassicae single-spore isolates with different virulence patterns. J. Phytopathol. 149:121-127.

Kuginuki, Y., Yoshikawa, H., and Hirai, M. 1999. Variation in virulence of Plasmodiophora brassicae in Japan tested with clubroot-resistant cultivars of Chinese cabbage (Brassica rapa L. ssp. pekinensis). Eur. J. Plant Pathol. 105:327-332.

Manzanares-Dauleux, M. J., Barret, P., and Thomas, G. 2000. Development of a pathotype specific SCAR marker in Plasmodiophora brassicae. Eur. J. Plant Pathol. 106:781-787.

Möller, M., and Harling, R. 1996. Randomly amplified polymorphic DNA (RAPD) profiling of Plasmodiophora brassicae. Lett. Appl. Microbiol. 22: 70-75.

Peng, Y. L., Huang, Y., Ye, L., Liu, Z. L., Yang, H., and Shang, J. 2016. Occurrence and morphology of small spheroid galls on clubroot-resistant Chinese cabbage. Eur. J. Plant Pathol. 145:591-599.

Schwelm, A., Fogelqvist, J., Knaust, A., Jülke, S., Lilja, T., Bonilla-Rosso, G., Karlsson, M., Shevchenko, A., Dhandapani, V., Choi, S. R., Kim, H. G., Park, J. Y., Lim, Ludwig-Müller, J., and Dixelius, C. 2015. The Plasmodiophora brassicae genome reveals insights in its life cycle and ancestry of chitin synthases. Sci. Rep. 5: Article 11153.

Sharma, K., Gossen, B. D., and McDonald, M. R. 2011. Effect of temperature on cortical infection by Plasmodiophora brassicae and clubroot severity. Phytopathology 101:1424-1432.

Shen, X. Q., Nie, K., Wu, Q., Zhang, Y. G., and Meng, X. H. 2009. Initial research report on differentiation identification of Chinese cabbage clubroot main physiological races. China Vegetables 2009(8):59-62.

Siemens, J., Keller, I., Sarx, J., Kunz, S., Schuller, A., Nagel, W., Schmülling, T., Parniske, M., and Ludwig-Müller, J. 2006. Transcriptome analysis of Arabidopsis clubroots indicate a key role for cytokinins in disease development. Mol. PlantMicrobe Interact. 19:480-494.

Somé, A., Manzanares, M. J., Laurens, F., Baron, F., Thomas, G., and Rouxel, F. 1996. Variation for virulence on Brassica napus L. amongst Plasmodiophora brassicae collections from France and derived single-spore isolates. Plant Pathol. 45:432-439.

Williams, P. H. 1966. A system for the determination of races of Plasmodiophora brassicae that infect cabbage and rutabaga. Phytopathology 56:624-626.

Yano, S., Tanaka, S., Ito, S., and Kameya-Iwaki, M. 1997. Variations of random amplified polymorphic DNA (RAPD) patterns among field populations of Plasmodiophora brassicae. Ann. Phytopathol. Soc. Jpn. 63:179-182.

Zhang, H., Feng, J., Manolii, V. P., Strelkov, S. E., and Hwang, S. F. 2015. Characterization of a gene identified in pathotype 5 of the clubroot pathogen Plasmodiophora brassicae. Phytopathology 105:764-770. 